\title{
Endoscopic posterior nasal neurectomy for intractable rhinitis: a systematic review of the literature Running Title: Endoscopic PNN: a systematic review
}

\author{
Mun Leng Lee ${ }^{1}$, Patrick Chakravarty ${ }^{2}$, and David Ellul ${ }^{1}$ \\ ${ }^{1}$ NHSGGC \\ ${ }^{2}$ Queen Elizabeth University Hospital
}

December 4, 2021

\begin{abstract}
Objectives: Rhinitis affects up to $40 \%$ of the population worldwide and can significantly reduce quality of life. Some patients remain symptomatic despite maximal medical therapy. In refractory cases, posterior nasal neurectomy (PNN - the division of the intranasal nerve branches containing postganglionic parasympathetic fibres) is postulated to reduce symptom burden. The objectives of this paper were to review the literature to establish whether the procedure is effective and safe in the management of allergic and non-allergic rhinitis. Design: A systematic review of Pubmed, EMBASE and MEDLINE was undertaken. Studies were excluded if not available in English or undertaken in non-human subjects. Participants: Seventeen articles satisfied the inclusion criteria studying in total 2029 patients. Sample size ranged from 8-1056. Main Outcome Measures: Patient-reported objective and subjective outcomes and post-operative complications were reviewed Results: There were two randomised controlled trials, two case control studies, and the remaining thirteen were case series using both objective and subjective outcome measures. All but one study found improved patient reported outcomes following PNN. Complications were reported in 10 studies - haemorrhage was the most common complication and was observed in 28 patients ( $1.6 \%$ of subjects). Conclusions: Endoscopic posterior nasal neurectomy is safe and appears to be effective in the treatment of intractable rhinitis but the level of the available evidence was generally poor. Larger, well designed studies are needed to clarify its role in the management of difficult-to-treat rhinitis.
\end{abstract}

\section{ABSTRACT}

Objectives: Rhinitis affects up to $40 \%$ of the population worldwide and can significantly reduce quality of life. Some patients remain symptomatic despite maximal medical therapy. In refractory cases, posterior nasal neurectomy (PNN - the division of the intranasal nerve branches containing postganglionic parasympathetic fibres) is postulated to reduce symptom burden. The objectives of this paper were to review the literature to establish whether the procedure is effective and safe in the management of allergic and non-allergic rhinitis.

Design: A systematic review of Pubmed, EMBASE and MEDLINE was undertaken. Studies were excluded if not available in English or undertaken in non-human subjects.

Participants : Seventeen articles satisfied the inclusion criteria studying in total 2029 patients. Sample size ranged from 8-1056.

Main Outcome Measures: Patient-reported objective and subjective outcomes and post-operative complications were reviewed.

Results: There were two randomised controlled trials, two case control studies, and the remaining thirteen were case series using both objective and subjective outcome measures. All but one study found improved 
patient reported outcomes following PNN. Complications were reported in 10 studies - haemorrhage was the most common complication and was observed in 28 patients (1.6\% of subjects).

Conclusions: Endoscopic posterior nasal neurectomy is safe and appears to be effective in the treatment of intractable rhinitis but the level of the available evidence was generally poor. Larger, well-designed studies are needed to clarify its role in the management of difficult-to-treat rhinitis.

Keywords: rhinitis, parasympathetic fibres, endoscopy

\section{KEYPOINTS}

- Rhinitis can have a significant negative impact on quality of life

- Medical management may not provide adequate relief

- Endoscopic posterior nasal neurectomy has been reported to improve symptoms

- We conducted a systematic review on endoscopic posterior nasal neurectomy

- Endoscopic posterior nasal neurectomy is safe and appears to be effective but further studies are required

\section{INTRODUCTION}

Rhinitis is an inflammatory condition of the nasal mucosa resulting in nasal obstruction, rhinorrhoea, sneezing and post-nasal drip. It is a global health problem and affects up to $40 \%$ of the population worldwide(1). It can have a significant impact on quality of life $(\mathrm{QoL})(2,3)$.

Management of rhinitis varies depending on the endotype and generally includes allergen or irritant avoidance, intranasal corticosteroid sprays, nasal saline rinses, oral or topical histamine-2 antagonists, ipratropium nasal sprays, leukotriene receptor antagonists, and immunotherapy. Turbinate surgery, vidian neurectomy, and more recently endoscopic posterior nasal neurectomy (PNN), are generally reserved for patients with disabling symptoms refractory to medical treatment.

Vidian neurectomy has been shown to effectively relieve the symptoms of both allergic and non-allergic rhinitis by dividing the preganglionic parasympathetic supply to the nasal mucosa $(4,5)$. However, it can result in xerophthalmia as the preganglionic fibres to the lacrimal gland are also inevitably divided during the procedure. Other complications include palatal and cheek numbness, ophthalmoplegia, and visual loss(46). PNN is a relatively recent surgical technique which avoids many of these complications, particularly xerophthalmia and maxillary nerve injury.

\section{Relevant anatomy and surgical technique}

The preganglionic parasympathetic fibres to the nasal mucosa leave the facial nerve as the greater superficial petrosal nerve and are joined by sympathetic fibres (deep petrosal nerve) to become the vidian nerve. This passes through the vidian canal and enters the pterygopalatine fossa (PPF) where the parasympathetic fibres synapse in the sphenopalatine ganglion (SPG). The posterior nasal nerve is a branch of the SPG within the PPF and consists of postganglionic parasympathetic, sympathetic, and sensory fibres. It enters the nasal cavity via the sphenopalatine foramen together with the sphenopalatine artery (SPA) and supplies the nasal mucosa providing a secretomotor and vasodilatory stimulus as well as somatic sensory supply(7-8). Two major branches of the posterior nasal nerve are often found anteroinferior and posterosuperior to the SPA but there are variations in the number and location of these branches(9). They are often overlooked during an SPA ligation as they are embedded in the connective tissue surrounding the artery. Division of these branches specifically targets the nerve fibres destined to innervate the nose and spares the fibres that innervate the lacrimal gland.

There are variations in the surgical technique of PNN but in general the procedure involves raising a mucosal flap off the sphenopalatine foramen and SPA and identifying the nerve and any major branches(9). These are divided and partly excised, and this can be achieved without dividing the SPA(11). PNN is a relatively

straightforward procedure, involves a less complicated dissection than vidian neurectomy(12), and can be carried out as a day case procedure. 
Rhinitis is a heterogenous condition with multiple overlapping endotypes and aetiologies(10). An imbalance between parasympathetic and sympathetic stimulation can potentially be a contributory mechanism. Dividing the posterior nasal nerve rather than the vidian nerve could potentially improve symptoms without the lacrimal and maxillary nerve complications seen frequently in vidian neurectomy(13). It also divides the somatosensory fibres which could further contribute to reducing nasal hypersensitivity to stimuli such as cold air(9-10).

\section{Objectives}

The objectives of this paper were to establish whether PNN is effective in reducing symptoms in allergic and non-allergic rhinitis and to review the safety profile of the procedure.

\section{MATERIALS AND METHODS}

A systematic review of the literature was undertaken to gather original data on rhinitis patients who underwent PNN. The Preferred Reporting Items for Systematic Reviews and Meta-Analyses (PRISMA) protocol was adhered to when applicable.

\section{Ethical considerations}

This systematic review did not require institutional ethics committee approval.

\section{Eligibility Criteria}

All published studies including case reports and interventional studies reporting original patient data were included. The inclusion criteria were: studies published in English, studies where PNN was performed either with or without additional procedures, and where measures of objective/subjective outcomes and/or complications were reported. Studies were excluded if they were unavailable in English, undertaken on animals, included patients with chronic rhinosinusitis with nasal polyps, or if other neurectomy procedures (eg vidian neurectomy) were performed instead of PNN.

\section{Information Sources \& Search Strategy}

A comprehensive literature search was undertaken using PubMed, EMBASE and MEDLINE. The search terms used were: "rhinitis AND surgical treatment", "rhinitis AND posterior nerve neurectomy", "rhinitis AND posterior nasal nerve", "rhinitis AND neurectomy", and "rhinitis AND nerve AND resection". The final search was carried out in July 2021. The primary outcomes of this study were the effectiveness (improvement in symptoms of rhinitis) and complications of PNN.

\section{Data Selection \& Collection Process}

All titles identified by the initial literature search were independently reviewed by two authors (LML and PDC) and the abstract and/or full text was reviewed where required. Data were extracted from papers that met the inclusion criteria by two authors. Extracted data included study type, sample size, surgical technique, study population characteristics, follow-up period, outcome measures, complications of surgery, and symptom outcomes. Any differences in extracted data were discussed and any disagreements resolved by the senior author (DE).

\section{Study Risk of Bias Assessment}

Two reviewers (PDC and DE) independently carried out an assessment of the quality of the yielded studies using the NIH Quality Assessment Tool(14).

\section{RESULTS}

An initial key word search yielded 2184 titles. Of these, 2090 were removed due to duplicate data and or irrelevant references. Ninety-four abstracts were deemed to be relevant and were reviewed in full. Of these, a further 68 studies were removed as they failed to meet the inclusion criteria. Seventeen studies with 2049 
subjects in total met the inclusion criteria and were included in this literature review. This process is shown in Figure 1.

\section{Study Characteristics}

The 17 papers that met the inclusion criteria are shown in Table 1 together with their quality assessment. These included two randomised controlled trials (RCTs). Zhang(15) compared the outcomes of PNN $(\mathrm{n}=$ 32 ) against nasal corticosteroid and oral antihistamines $(\mathrm{n}=31)$ and Hua et al $(16)$ compared the outcomes of PNN alone against PNN with pharyngeal neurectomy where the former group was used as the control group. There were two case-control studies $(17,18)$ - both comparing outcomes of turbinate reduction with and without PNN. The remaining thirteen $(9,12-13,19-28)$ were case series; 5 prospective and 8 retrospective. Ogi et al.(25) published a case series and undertook a case-control sub-analysis on complications by sending questionnaires to patients who underwent endoscopic sinus surgery (without PNN) as a control. Five studies $(12,15,23-24,26)$ examined PNN as a single intervention with no concomitant nasal surgery. Eight studies $(9,13,19-22,27-28)$ examined PNN with concurrent turbinate and septal surgery and four $(17-18,21,25)$ examined PNN with concurrent turbinate reduction.

Two studies $(9,17)$ specified that entire PNN was not undertaken and only posterior nasal nerve branches within the inferior turbinate or middle meatus were divided to improve symptoms. Kobayashi(21) retrospectively compared patients who underwent selective PNN to total PNN. The SPA was divided together with the posterior nasal nerve in four studies $(15,25,26,28)$.

Sample sizes ranged from 8 to 1056 patients. The follow up period ranged from six months to eight years. Five studies $(12,17,19-20,22)$ cited a single post-operative review with no mention of timing, whereas Kanaya et al $(12,22)$ did not specify their follow up at all.

Ten studies $(9,13,15-18,21-22,25,27)$ recruited patients with allergic rhinitis only and three studies $(19,20,28)$ included both allergic and non-allergic patients. The allergy status was not recorded in 4 studies(12,23-24,26). Allergic rhinitis was defined by IgE or RAST testing in six studies $(17,18,19,20,25,28)$, skin prick testing in one study $(27)$, and diagnosis was confirmed by both in two studies $(9,13)$. The remaining studies did not define how allergic rhinitis was diagnosed in the study populations. Eleven studies $(9,13,15-16,18-19,22-25,28)$ specified the treatments that had been used prior to PNN.

\section{Result of Individual Studies}

Outcome measures used and results are shown in Table 2. In one study(12), the outcome measure used was not specified - 94 of their cohort of 1056 patients were sent a questionnaire 2 years following the procedure but no further detail was provided. The authors of this study reported an $80 \%$ efficacy rate after 2 years.

The RCT(15) comparing PNN with medical treatment (corticosteroids and antihistamines for 3 months) utilised the RQLQ, a VAS for pain, and the Schirmer's test before and after the intervention (at 1, 3, 6 and 12 months). RQLQ scores were significantly better in the PNN group at 1, 3, 6, and 12 months following surgery when compared to the control group. There was no significant difference in the Schirmer's test outcome in either group before and after treatment. They also reported a significant improvement in pain (the site and type of pain was not specified) in the PNN group at 1, 3, 6, and 12 months.

The second RCT(16) compared a group undergoing PNN combined with pharyngeal neurectomy with a control group undergoing PNN only. The authors reported a significant improvement in RQLQ and VAS scores up to 24 months post intervention in both groups with no significant difference between them.

Suzuki et al(17) analysed outcomes in patients undergoing turbinoplasty with and without PNN. They found a significant reduction in sneezing and rhinorrhoea and olfactometry after turbinate reduction with PNN that was not seen in the turbinate reduction group. Albu et al(18) undertook a similar study in patients with severe inferior turbinate hypertrophy comparing outcomes after turbinate reduction with and without PNN. Subjective and objective outcome parameters suggested improvement in both study groups with no differences between the two. 
In the case series analysis, three papers $(23,24,26)$ studied PNN as a single intervention and found a significant improvement in patient reported symptoms (SNOT-22, RQLQ, and individual nasal symptom scores)with follow-up ranging from 6 to 12 months.

The other case series $(9,13,18,20-21,27-28)$ looked at outcomes of PNN with turbinate reduction and/or septoplasty. They all reported significant subjective improvements in rhinitis symptoms and quality of life (when this was assessed). In this group only Ikeda et al.(19) studied objective measures and found significant improvement in rhinomanometry and nasal provocation following surgery.

\section{Complications}

Complication data are shown in Table 3 . Across the 15 studies where complications were recorded, a total of 60 patients experienced complications giving an overall rate of $3 \%$.

\section{Haemorrhage}

Post-operative haemorrhage was reported as a complication in 8 studies(12,16,18-19,21,25-27) with a total of 28 patients in the PNN arms giving an overall haemorrhage rate of $1.6 \%$. The haemorrhage rates in these studies were $0.006 \%(12), 1.78 \%(19), 3.9 \%(25,27), 9 \%(21)$, and $28 \%(18)$ with return to theatre rates ranging from $0.006 \%(12)$ to $2.4 \%(25)$.

\section{Other complications}

Six studies $(13,19,21,25,27-18)$ cited non-haemorrhagic complications. Sensory disturbance or numbness of the lip, palate or teeth was the most common and affected 32 patients in these studies - this was transient in 17 patients(9.7\% of patients in relevant studies), and permanent in $7(4 \%)$ patients. Wang $(27)$ and Sonoda(28) reported lip, oral, or pharyngeal numbness in 8 patients $(4.6 \%)$ but did not specify whether this was permanent or transient. Sonoda(28) reported that all their adverse events including numbness were mild.

Ogi et al.(25) reported 7 patients in their PNN group having persistent numbness, but this was comparable to the percentage of patients in their control group who underwent endoscopic sinus surgery (ESS) without PNN. The sensory disturbance was classified as mild in 4 patients and severe in 3 patients.

Two papers reported post-operative adhesions in $23.5 \%(23)$ and $6.5 \%(27)$ of patients.

\section{DISCUSSION}

The principal finding of this literature review was that PNN is a safe procedure with an acceptable complication rate. It appears to be an effective procedure for patients with allergic and non-allergic rhinitis but the level, quality, and heterogeneity of the published evidence hampers the strength of the conclusions that can be drawn. Every study but one reported symptom improvement in patients undergoing PNN. Outcome measures were largely valid and improvements were observed in all individual nasal symptoms (particularly sneezing and rhinorrhoea) as well as QoL measures, but most studies did not have a control arm.

\section{Complication rates}

Bleeding was the most common complication of the procedure and affected $1.6 \%$ of patients. In the studies reporting higher rates, all patients underwent turbinate reduction in addition to PNN. Sensory disturbance of the palate or lip was also observed as a complication, but this was noted to be usually transient. Ogi(25) was the only study that reported permanent numbness. Interestingly the authors reported similar rates of persistent numbness in the control group that underwent ESS without PNN. Their surgical procedure involved turbinate reduction using an incision anterior to the anterior pole of the inferior turbinate (at the level of the pyriform aperture) where terminal branches of the infraorbital nerve might be found on the anterior surface of the maxillary bone. Indeed Sonoda(28) also noted that it is likely to be the turbinate reduction or septoplasty component of the surgery that was likely responsible for dental or palatal numbness. Only 1 patient across all the studies reported persistent mild dryness of the eyes following the procedure but this did not require any treatment(28). 


\section{Effectiveness}

The RCT in patients with persistent, moderate-severe allergic rhinitis(15),found that PNN resulted in an improved VAS score for pain and improved RQLQ scores at 1, 3, 6, and 12 months following the surgery compared with patients treated medically. The quality and site of the pain was not reported. There was no objective change in tearing/watery eyes in either group following intervention. One major limitation of this study was the duration of medical treatment in the control group - these patients only received intranasal corticosteroid spray (INCS) and an oral antihistamine for 3 months and therefore the improved scores in the PNN group beyond 3 months were measured at a time when the control group was not receiving any treatment whatsoever. One could also argue that INCS and an oral antihistamine alone do not constitute "maximal medical therapy" for allergic rhinitis and all this, together with the small sample size reduced the quality of this study and the conclusions that can be drawn.

The second RCT(16) compared PNN to PNN with pharyngeal neurectomy and therefore the focus was on assessing whether the pharyngeal neurectomy contributed to symptom control. Therefore, this study design did not allow us to draw any robust conclusions as to the effectiveness of PNN itself. Both groups had significant improved outcomes up to 2 years following the intervention.

Two case control studies $(17,18)$ directly compared patients who underwent turbinate reduction with and without PNN. Sample size, methodology and operative approach were similar in both studies. Albu(18) found no significant difference in VAS scores, RQLQ scores and rhinomanometry in patients who underwent PNN in addition to turbinate reduction. The only significant difference was an increased likelihood of post-operative haemorrhage in the PNN group. This study recruited (retrospectively) patients with severe inferior turbinate hypertrophy with allergic rhinitis and the predominant pre-operative symptom was nasal obstruction although rhinorrhoea, post-nasal drip, snoring, and sneezing were also present and all improved in both arms with no significant differences between the two. There was a larger improvement in rhinorrhoea in the turbinoplasty + PNN group but this was not statistically significant. One must bear in mind that terminal branches of the posterior nasal nerve within the inferior turbinate are potentially divided during a turbinoplasty. Suzuki(17) found significant improvement in the PNN group in VAS scores for sneezing, nasal obstruction and nose blowing, and improvement in olfaction using olfactometry. The risk of bias was deemed to be high in both studies.

Six studies(13,16,18-19,25,28) followed up patients for longer than 12 months. Of the studies that followed up at three years or greater, it would appear that benefits may diminish over time(25,28). Ogi et al.(25) reported that improvements in sneezing, rhinorrhoea and obstruction were no longer present after 6 years. Sonoda et al.(28) found that significant improvements in rhinorrhoea and sneezing had diminished at 8 years, but the improvement in nasal obstruction, JRQLQ, TNSS scores, and reduction in medication use was sustained over eight years. It was not possible with the data available from the various studies to infer whether patients with allergic backgrounds had a different outcome to those with non-allergic backgrounds.

Apart from 4 studies(15-18), the evidence was level 4. There was a high risk of bias and confidently attributing the outcomes to PNN was not possible. Follow up was quite variable and frequently timing of follow up was not cited. Study design varied considerably and a mixture of different outcome measures made the data too heterogeneous to undertake a meta-analysis. More than half of the subjects were yielded from the Kanaya case series(12). This study did not specify their patient population in terms of rhinitis status, previous treatment, follow up period, and symptomatic outcomes. Almost all studies were carried out in East and South Asian patients and this must be borne in mind when assessing the applicability of the results to Western populations.

\section{CONCLUSIONS}

To our knowledge this is the first literature review specifically focussing on PNN and our review encompassed the entire history of the procedure back to 1997. We undertook a robust search which yielded enough studies to undertake a meaningful evaluation of the procedure to date in patients with both allergic and non-allergic rhinitis. 
PNN is a safe procedure with a low risk of complications. The available literature suggests it is effective in controlling symptoms and all studies but one reported significant symptomatic improvement following PNN with or without other procedures. The procedure should be considered in rhinitic patients with a high symptom burden inspite of maximal medical therapy. However, there is a need for high quality, adequately powered, randomised controlled trials with longer follow-up periods comparing patient-reported outcomes after PNN to medical therapy.

\section{References}

1. Klementina S, Avdeeva, Fokkens W, Reitsma S. Towards a new epidemiological definition of chronic rhinitis: prevalence of nasal complaints in the general population. Rhinology. 2021 June; 59(3): 258-266.

2. Wallace DV, Dykewicz MS, Bernstein DI, Blessing-Moore J, Cox L, Khan DA, et al. The diagnosis and management of rhinitis: an updated practice parameter. J Allergy Clin Immunol. 2008 Aug;122(2 Suppl):S1-84. doi: 10.1016/j.jaci.2008.06.003. Erratum in: J Allergy Clin Immunol. 2008 Dec;122(6):1237.

3. Price D, Scadding G, Ryan D, Bachert C, Canonica W, Mullol J, et al. The hidden burden of adult allergic rhinitis: UK healthcare resource utilisation survey. Clin Transl Allergy. 2015;5:39

4. Robinson SR, Wormald PJ. Endoscopic vidian neurectomy. Am J Rhinol. 2006;20(2):197-202.

5. Lee JC, Kao CH, Hsu CH, Lin YS. Endoscopic transsphenoidal vidian neurectomy. Eur Arch Otorhinolaryngol. 2011 (Jun); 268(6):851-856.

6. El-Guindy A. Endoscopic transseptal vidian neurectomy. Arch Otolaryngol Head Neck Surg. 1994 Dec; 120(12):1347-1351.

7. Baraniuk JN, Lundgren JD, Okayama M, Mullol J, Merida M, Shelhamer JH, Kaliner MA. Vasoactive intestinal peptide in human nasal mucosa. J Clin Invest. 1990 Sep;86(3):825-31.

8. Osawa S, Rhoton AL Jr, Seker A, Shimizu S, Fujii K, Kassam AB. Microsurgical and Endoscopic Anatomy of the Vidian Canal. Neurosurgery 2009; 64:385-411.

9. Takahara D, Takeno S, Hamamoto T, Ishino T, Hirakawa K. Management of Intractable Nasal Hyperreactivity by Selective Resection of Posterior Nasal Nerve Branches. International Journal of Otolaryngology. 2017; 2017:1907862

10. Papadopoulos N, Guibas G. Rhinitis Subtypes, Endotypes, and Definitions.Immunol Allergy Clin North Am. 2016 May; 36(2): 215-233.

11. Kawamura S, Asako M, Momotani A, Kedai H. Submucosal Turbinectomy with Posterior-Superior Nasal Neurectomy for Patients with Allergic Rhinitis. Practica Oto-Rhino-Laryngologica.2000; 93(5):367-372.

12. Kanaya T, Kikawada T. Endoscopic posterior nasal neurectomy: an alternative to Vidian neurectomy. Operative Techniques in Otolaryngology.2009;9:24-27.

13. Ogawa T, Takeno S, Ishino T, Hirakawa K. Submucous turbinectomy combined with posterior nasal neurectomy in the management of severe allergic rhinitis: Clinical outcomes and local cytokine changes. Auris Nasus Larynx 2007;34: 319-326.

14. NIH National Heart, Lung, and Blood Institute Quality Assessment Tool. Study Quality Assessment Tools. [Cited on 15th July 2021]. Available from: https://www.nhlbi.nih.gov/health-topics/studyquality-assessment-tools.

15. Zhang Y, Ma D, Xu K, Feng Y, Sun J, Zhu Q. Treating moderate-to-severe persistent allergic rhinitis by endoscopic posterior nasal neurectomy: a study of efficacy and effects on autophagy.International Journal of Clinical and Experimental Medicine.2020;13(7):4864-4871

16. Hua H, Wang G, Zhao Y, Wang D, Qiu Z, Fang P. The long-term outcomes of posterior nasal neurectomywith or without pharyngeal neurectomy in patientswith allergic rhinitis: a randomized controlled trial. Brazilian Journal of Otorhinolaryngology. 2021;1808-8694(21)00091-4.

17. Suzuki M, Yokota M, Ozaki S, Murakami S. The effects of resection of the peripheral branches of the posterior nasal nerves in the inferior turbinate, with special focus on olfactory dysfunction. The Journal of laryngology and otology.2019;133(12):1046-1049. 
18. Albu S, Trombitas V, Nagy A. Endoscopic microdebrider-assisted inferior turbinoplasty with and without posterior nasal neurectomy. Auris, nasus, larynx. 2014; 41(3):273-277.

19. Ikeda K, Oshima T, Suzuki M, Suzuki H, Shimomura A. Functional inferior turbinosurgery (FITS) for the treatment of resistant chronic rhinitis. Acta oto-laryngologica. 2006; 126(7):739-745.

20. Ikeda K, Yokoi H, Saito T, Kawano K, Yao T, Furukawa M. Effect of resection of the posterior nasal nerve on functional and morphological changes in the inferior turbinate mucosa. Acta oto-laryngologica. 2008;128(12):1337-1341.

21. Kobayashi T, Hyodo M, Nakamura K, Komobuchi H, Honda N. Resection of peripheral branches of the posterior nasal nerve compared to conventional posterior neurectomy in severe allergic rhinitis. Auris, nasus, larynx. 2012;39(6):593-596.

22. Kanaya T, Kohno N. Endoscopic Posterior Nasal Neurectomy with Continuous-Suction Irrigation Method. Journal of Otolaryngology Research. 2017;1(1):113.

23. Arun G, Sanu M, Mohan M, Aparna T, Afroze K. Effectiveness of endoscopic posterior nasal neurectomy for the treatment of intractable rhinitis. Romanian Journal of Rhinology. 2017; 7(26):85-90.

24. Vo MC, Pham HK, Nguyen MH. Posterior Nasal Neurectomy in Treatment of Intractable Rhinitis: A Preliminary Series. Philippine Journal of Otolaryngology Head and Neck Surgery. 2018;33(1):12-16.

25. Ogi K, Manabe Y, Mori S, Kimura Y, Tokunaga T, Kato Y, et al. Long-Term Effects of Combined Submucous Turbinectomy and Posterior Nasal Neurectomy in Patients with Allergic Rhinitis. SN Comprehensive Clinical Medicine. 2019;1: 540-546.

26. Ahilasamy N, Dinesh K. Endoscopic posterior nasal neurectomy. The Journal of laryngology and otology. 2019;133(9):1-5.

27. Wang L, Chen M, Xu M. Effect of posterior nasal neurectomy on the suppression of allergic rhinitis. American journal of otolaryngology.2020;41(3):102410.

28. Sonoda S, Murakami D, Saito Y, Miyamoto Y, Higuchi R, Kikuchi Y, Sawatsubashi M, Nakagawa T. Long-term effectiveness, safety, and quality of life outcomes following endoscopic posterior nasal neurectomy with submucosal turbinectomy for the treatment of intractable severe chronic rhinitis. Auris, nasus, larynx. 2021;48(4):636-645

\section{Hosted file}

Figure 1 - Flow chart showing literature search strategy.docx available at https: //authorea.com/users/449501/articles/548023-endoscopic-posterior-nasal-neurectomy-forintractable-rhinitis-a-systematic-review-of-the-literature-running-title-endoscopic-pnna-systematic-review

\section{Hosted file}

Final Table 1 - Sample size, study design, population studied, interventions and follow up in the studi available at https://authorea.com/users/449501/articles/548023-endoscopic-posterior-nasalneurectomy-for-intractable-rhinitis-a-systematic-review-of-the-literature-running-titleendoscopic-pnn-a-systematic-review

\section{Hosted file}

Final Table 2 - Outcome measures by study.docx available at https://authorea.com/users/ 449501/articles/548023-endoscopic-posterior-nasal-neurectomy-for-intractable-rhinitis-asystematic-review-of-the-literature-running-title-endoscopic-pnn-a-systematic-review

\section{Hosted file}

Final Table 3. Complications recorded in the seventeen studies included..docx available at https://authorea.com/users/449501/articles/548023-endoscopic-posterior-nasal-neurectomyfor-intractable-rhinitis-a-systematic-review-of-the-literature-running-title-endoscopicpnn-a-systematic-review 